\title{
DEL RECURSO AL TELETRABAJO COMO MEDIDA DE EMERGENCIA AL FUTURO DEL TRABAJO A DISTANCIA
}

\author{
From telework as an emergency measure to the future of remote work \\ Henar Álvarez Cuesta* \\ Universidad de León, España
}

\begin{abstract}
RESUMEN
La situación derivada de la declaración del estado de alarma provocado por la pandemia causada por el COVID19 supuso un verdadero terremoto en el mundo laboral, uno de cuyos efectos secundarios ha sido la conversión «mágica» del trabajo presencial a teletrabajo y todo parece indicar que su uso se difundirá con mayor intensidad en el futuro. Por tal motivo, conviene analizar los puntos débiles de la regulación actual del trabajo a distancia, capaces de impedir un trabajo que merezca el calificativo de digno; proponer los contenidos necesarios a reformular, como la protección de la salud y seguridad, el control empresarial, la interconexión con el trabajo flexible o las brechas de género que puede desencadenar; y, por último, avanzar las vías para su aplicación en cada organización.

Palabras clave: teletrabajo, flexibilidad, acuerdo, control empresarial, conciliación.
\end{abstract}

\section{ABSTRACT}

The situation derived from the declaration of the state of alarm caused by COVID19 supposed a real earthquake in the world of work, one of whose side effects has been the "magical" conversion of traditional work to telework and everything seems to indicate that telework will spread more intensely in the future. For this reason, it is convenient to analyze the weak points of the current regulation of remote work; to propose the necessary contents to reformulate, such as the protection of health and safety, business control, the interconnection with flexible work or the gender gaps; and, finally, to advance the ways for its application in each organization.

Keywords: telework, flexibility, agreement, business surveillance; work-family balance.

\footnotetext{
* Correspondencia a: Henar Álvarez Cuesta. Facultad de Ciencias del Trabajo, Universidad de León, Campus de Vegazana, s/n, 24071-León (España) - halvc@unileon.es - https://orcid.org/0000-0003-0957-1515

Cómo citar: Álvarez Cuesta, Henar. (2020). «Del recurso al teletrabajo como medida de emergencia al futuro del trabajo a distancia»; Lan Harremanak, 43, 175-201. (https://doi.org/10.1387/lan-harremanak.21722).

Recibido: 14 mayo, 2020; aceptado: 14 junio, 2020.

ISSN 1575-7048 - eISSN 2444-5819 / (c) 2020 UPV/EHU
}

(c) (i) Esta obra está bajo una licencia

Creative Commons Atribución 4.0 Internacional 


\section{Concepto}

Muchas veces se emplean de forma indistinta los conceptos de teletrabajo, trabajo a distancia y trabajo en el domicilio. En principio, el teletrabajo es trabajo a distancia, pero no todo trabajo a distancia es teletrabajo (prestación de servicios mediante el uso intensivo de la tecnología).

El teletrabajo es solo una parte del género que constituye el trabajo a distancia (Purcalla Bonilla y Preciado Domènech, 2013: 486). Así, es posible encontrar trabajo a distancia que no incluya teletrabajo, y de igual modo, existe trabajo con dispositivos tecnológicos que no supone trabajo a distancia.

También surgen o se definen otras categorías, como el teletrabajo invisible o el «smart work». Respecto al primero, más que una categoría cabría considerarlo un riesgo laboral, en tanto es aquel que «se produce de manera no organizada como una continuación de la jornada ordinaria de trabajo en el domicilio del trabajador, sin control ni remuneración» (Rodríguez-Piñero Royo, Miguel, 2018: 93), cuyo último paso sería la desaparición (en falso) de la propia laboralidad de la prestación al quedar extramuros de la regulación laboral (trabajo sumergido).

Por su parte, se ha comenzado a denominar smart work a aquella prestación de servicios con los siguientes rasgos distintivos: movilidad permanente, comunicación continua y bilateral trabajador/empresa, pluralidad de lugares de trabajo, alto nivel de conectividad, trabajo remoto, acceso global y constante al conocimiento, networking, autogestión del tiempo de trabajo y horarios y jornadas de trabajo flexibles (Rodríguez-Piñero Royo, Miguel, 2018: 93); en suma, teletrabajo combinado con prestación presencial de forma flexible.

La característica principal de todos estos modos y maneras de trabajar viene dada en negativo: la ausencia del centro de trabajo como lugar de prestación de servicios, desarrollándose esta bien en el domicilio (conocido como SOHO por sus siglas en inglés, smal office, home office), bien en otro lugar libremente elegido por este o en lugares variables, itinerantes o nómadas, precisados o precisables, cuando así responda al interés de las partes (Mella Méndez, 2002: 7). En esta definición, y teniendo en cuenta la situación actual, se ha de tener también en cuenta las restricciones a la movilidad, desarrollándose en este momento la prestación en la residencia de la persona trabajadora, no exigiéndose que sea aquella la formal sino la realmente utilizada.

De atender propiamente al teletrabajo, la multiplicidad y la heterogeneidad de sus modalidades permite acudir a él con flexibilidad (e imaginación), pero cabe advertir ya desde el comienzo que puede acabar desencadenando la huida (o disfraz) del trabajo por cuenta ajena hacia el empleo sumergido o falsamente autónomo. 
En los casos más extremos de desvinculación, pueden darse prestaciones de servicios en las que incluso se pueda poner en tela de juicio la propia laboralidad de la misma, en cuyo caso resultará menester acudir —en este como en tantos otros supuestos, por ejemplo, el trabajo a través de plataformas digitales (Álvarez Cuesta, 2018) - al necesario análisis casuístico de las circunstancias que rodean dicha prestación.

Quedan fuera de esta categoría de trabajo a distancia las actividades intrínsecamente externas, ya sean de carácter fijo o se desarrollen de manera itinerante. Igualmente deben quedar excluidas aquellas $s$ que no se prestan fuera de la empresa, como contact center (García Romero, 2012: 45) aun cuando se realicen on line en el centro de trabajo.

\section{El teletrabajo en España, entre la tradición del presentismo y las ventajas on line}

La situación provocada por el COVID19 ha configurado el recurso al teletrabajo como la gran solución (con aristas, desde luego, tanto para el empleo privado como el público) para el futuro de la actividad económica (al menos en parte).

Y lo ha hecho en un país donde el teletrabajo aún es una realidad incipiente y en franco crecimiento. Es verdad que no ha encontrado un desarrollo masivo y todavía pasa por ser una posibilidad minoritaria y, en buena medida exótica, en un tejido productivo aún ampliamente forjado sobre la nota (que hoy ya debería haber quedado obsoleta) de presencialidad, cuando no un más pernicioso presencialismo (De Las Heras García, 2016: 43) y todo ello a pesar de la valoración positiva, como "forma de modernizar la organización del trabajo», que realiza el punto 4 del Capítulo II del II Acuerdo para el Empleo y la Negociación Colectiva de 2012 (reiterado en el III Acuerdo de 2015).

Tradicionalmente, el teletrabajo no se ha sido acogido en España con el mismo interés y la misma aplicación que en otros países de nuestro entorno, quizá por concurrir en la cultura empresarial española el mal antes citado - la implantación de colectivos de trabajadores asalariados con dispositivos digitales que trabajen fuera de la empresa, ya sea como teletrabajadores, ya sea como trabajadores móviles, es reducido, ya que solo se cuenta el 6,7 \% de los empleados frente al 17 \% de la Unión Europea (Camas Roda, 2018: 189)—.

Según los últimos estudios, respecto al uso del teletrabajo por los españoles, el $19 \%$ de los españoles y el $21 \%$ de los europeos teletrabajaron al menos una vez en 2018. Así destaca Reino Unido, con un porcentaje de 33\% de individuos 
que teletrabajaron al menos una vez, mientras Francia (23\%) y Alemania (22\%) también tiene valores por encima de España. El 17\% de los españoles utilizaron Internet cuando teletrabajaban, por debajo de la media de la UE 28 (20\%), y de Reino Unido (32\%), Francia (20\%), Alemania (20\%), pero por encima de Italia (12\%). La mayor proporción de individuos de las principales economías europeas que teletrabajan al menos una vez a la semana se da en Reino Unido (20\%), Francia (16\%), Alemania (13\%) y UE (13\%) y por debajo se encuentra España (12\%) e Italia (9\%) (ONTSI, 2020: 4).

En consecuencia, la realidad en Espańa contrasta con cuanto ocurre en los tejidos productivos de los países de nuestro entorno, donde se han valorado positivamente las potencialidades que presenta esta modalidad laboral, y, en tasa creciente, el porcentaje de operarios que prestan sus servicios - de forma total o, más frecuentemente, parcial — se ha incrementado notablemente (Sanguinetti Raymond, 2003: 19).

Las ventajas a las que se ha aludido supra son de todos conocidas. Así, el teletrabajo aparece asociado con una mayor productividad: por ejemplo, en Suecia, casi el $80 \%$ de los empleadores declararon que permitir trabajar en ciertas ocasiones fuera de las instalaciones conducía a mayor productividad, debido a que los teletrabajadores son interrumpidos con menos frecuencia por colegas o sus superiores, pasan menos tiempo contestando llamadas telefónicas o correo electrónico y no tienen que moverse hacia y desde la oficina (Eurofound y OIT, 2017: 26).

Al tiempo, la ausencia de desplazamientos laborales reduce el tráfico, los accidentes in itinere y como consecuencia añadida disminuye la contaminación ambiental y las emisiones con efecto invernadero de conformidad con la Carta Europea del Teletrabajo.

El teletrabajo puede ser también una vía para atraer el talento de quienes ostentan una mayor cualificación y retener a los trabajadores, reducir el estrés (Eurofound y OIT, 2017: 26) y aumentar su motivación e implicación en la empresa (Thibault Arana, 2000: 41).

Otra de las ventajas que conlleva el teletrabajo, hasta el punto de constituir una de las posibilidades destinadas a facilitar la conciliación de la vida laboral, personal y familiar, es su contribución al equilibrio entre el trabajo y la vida (en Francia el 95\% de los encuestados declaró que el teletrabajo ha tenido un impacto positivo en su calidad de vida tanto en el trabajo como fuera de ella; $89 \%$ reportaron una mejor calidad de vida familiar; y el $88 \%$ percibió un mejor equilibrio entre el trabajo y la vida). Sin embargo, y como se analizará en profundidad a la hora de examinar los riesgos laborales, el $61 \%$ de las personas que teletrabajan reconocen trabajar más horas porque se confunden los tiempos de trabajo y descanso, la jornada se vuelve «elástica». También hay 
diferencias importantes por grupos de trabajadores según el género, por ejemplo, las mujeres tienden a trabajar menos horas y parecen tener resultados ligeramente mejores en conciliar el trabajo y la vida que los hombres (Eurofound y OIT, 2017: 26).

Por último, esta forma de realizar las tareas a distancia acaba por facilitar la organización flexible de los recursos empresariales, propiciando el ahorro en costes (NTP 412); puede coadyuvar a la revitalización de zonas rurales en riesgo de despoblación; e incluso permitir mejorar la empleabilidad de determinados colectivos de trabajadores que por sus características personales (edad, discapacidad, víctima de violencia de género...) tendrían difícil acceder a un puesto presencial (Lousada Arochena, 2011: 299).

Por todo ello, y sobre todo por la necesidad de proteger la salud de los trabajadores y de la sociedad en su conjunto en las nuevas coordenadas sanitarias y sociales, parece que el teletrabajo (al menos algunas de sus modalidades, pues cabe augurar que algunas modalidades como el hot desking no tendrán cabida) va a experimentar un rápido crecimiento y una expansión sin precedentes. Con todo, no cabe pasar por alto los riesgos (algunos ya apuntados y otros desarrollados en las siguientes páginas) que conlleva su aplicación sin haber definido y acordado sus parámetros.

\section{Regulación «oculta» del teletrabajo}

A nivel internacional (OIT, 2020) se ha elaborado el Convenio núm. 177 de la OIT (Rojo, 2020b), sobre trabajo a domicilio y la Recomendación núm. 184 OIT, aún no ratificado por España, el cual estableció unos niveles de protección mínimos para todos los trabajadores cuya característica principal residía en la no coincidencia espacio/temporal con el empleador (Espín Sáez, 2014: 249).

En la Unión Europea se aprobó el Acuerdo Marco Europeo sobre Teletrabajo (AMET), firmado el 16 de julio de 2002 en Bruselas por los agentes sociales (CES, UNICE/UEAPME y CEEP), verdadero revulsivo en cuanto a la visibilidad y ordenación de la institución. Con todo, este Acuerdo no compromete directamente a los Estados, sino a las propias organizaciones empresariales de trabajadores firmantes, quienes se obligan a poner en marcha el pacto en los Estados miembros, conforme a los procedimientos y prácticas específicas de cada uno de ellos. En España no se ha llevado a cabo una incorporación "real y efectiva» al ordenamiento jurídico español (STS 11 abril 2005 Rec. núm. 143/2004), en tanto se incluyó como anexo al Acuerdo Interconfederal para la Negociación Colectiva de 2005. Con todo, constituye un referente ineludible para los agentes sociales y para la doctrina científica (Serrano García, 
2002: 34), en tanto los primeros lo consideran «un instrumento especialmente útil para facilitar y extender la introducción del teletrabajo en las empresas y resolver algunas dudas que venían surgiendo en determinados aspectos del campo laboral en relación con el teletrabajo» (Acuerdo Interconfederal para la Negociación Colectiva de 2003). Los sucesivos Acuerdos han llevado a cabo una "tímida» regulación del mismo (CC.OO., 2020b: 23).

En la regulación interna, el precepto que diseña su régimen jurídico de forma imprecisa y parca (Leonés Salido, 2000: 2) el art. 13 ET, no habla del teletrabajo, sino de la categoría más amplia del trabajo a distancia, definiéndolo como aquel que «en que la prestación de la actividad laboral se realice de manera preponderante en el domicilio del trabajador o en el lugar libremente elegido por este, de modo alternativo a su desarrollo presencial en el centro de trabajo de la empresa» (art. 13.1).

La posibilidad de que el trabajador desempeñe sus tareas profesionales para el empresario fuera del espacio físico constituido por el centro de trabajo ha sido reconocida tradicionalmente por el Derecho del Trabajo, sometiendo tal circunstancia a determinadas cautelas adicionales para evitar que la falta de contacto físico entre operario y empleador pudiera suponer abusos o arbitrariedades en el desempeño de la prestación (Solà i Monels, 2004: 211).

La tecnología (su uso y difusión) ha cambiado (y seguirá haciéndolo) los modos y maneras de trabajar, además, la crisis (sanitaria, social y económica) provocada por el COVID19 ha modificado decisivamente la perspectiva de la posibilidad de prestación laboral sin coincidencia en el mismo espacio físico, ni entre trabajadores ni con su empleador (Sempere Navarro y Kahale Carrillo, 2013).

A la vista de la posible inercia en el sistema de relaciones laborales y la configuración del sistema productivo español integrado por pequeñas empresas, resulta cada vez más necesario diseñar un Acuerdo Marco interprofesional que fije más detalladamente los parámetros para hacer efectivo un teletrabajo digno y de calidad, con espacio para que la negociación colectiva sectorial desarrolle las coordenadas a cada actividad productiva y sin perjuicio de mejoras o adaptaciones introducidas por los convenios de empresa, siempre y cuando respeten los mínimos contenidos en los referentes mentados (cabe dar algunos ejemplos relevantes, como la regulación realizada en el II Convenio Colectivo de empresas vinculadas para Telefónica de España, SAU, Telefónica Móviles España, SAU y Telefónica Soluciones de Informática y Comunicaciones de España, SAU, BOE núm. 273, de 13 de noviembre de 2019). 


\section{Requisitos necesarios para teletrabajar}

De poner el foco en la prestación de servicios realizada a través de la tecnología (teletrabajo) aun proyectadas para todo el trabajo a distancia por el art. 13 ET, conviene analizar las condiciones requeridas para su aplicación:

A. Forma escrita. Es necesario que la prestación de servicios a distancia mediante el uso de la tecnología se formalice por escrito (siempre ad probationem, nunca ad solemnitatem). Aun cuando no establece cuál habrá de ser la consecuencia de la falta de forma, cabe entender que la consideración de la prestación como debida en el centro de trabajo al que esté adscrita la persona trabajadora.

El precepto examinado recoge expresamente la obligación de aplicar las reglas referidas a la copia básica del contrato de trabajo, garantía en orden al control del cumplimiento de la normativa laboral.

B. Voluntario. La voluntariedad exigida en el teletrabajo supone la necesidad de contar con el acuerdo de ambas partes para el desarrollo de la prestación de servicios en un lugar diferente. Dicho acuerdo puede nacer al inicio de la relación laboral (con la oferta de trabajo bajo tal característica y la persona trabajadora aceptará la misma junto con las restantes condiciones) o bien durante su transcurso. En consecuencia, cabe realizarlo posible en cualesquiera de las modalidades contractuales previstas a lo largo y ancho del ordenamiento laboral — contrato a tiempo parcial incluido (Serrano García, 2009: 207)—, como un contenido accesorio al mismo (Tascón López, 2020).

Tanto el art. 3 del Acuerdo Marco Europeo como el art. 6 del Convenio 177 OIT imponen las notas de libertad y voluntariedad para las partes, sin que la persona trabajadora pueda sufrir ninguna consecuencia desfavorable por negarse a aceptar su realización. Así, el Acuerdo Europeo reconoce cómo «el teletrabajo es voluntario para el trabajador y el empresario afectados. El teletrabajo puede formar parte de la descripción inicial del puesto de trabajo o puede incorporarse de forma voluntaria más tarde... Si el teletrabajo no forma parte de la descripción inicial del puesto, y si el empresario hace una oferta de teletrabajo, el trabajador puede aceptarla o rechazarla. Si es el trabajador quien expresa su deseo de teletrabajar el empresario puede aceptarla o rechazarla. El paso al teletrabajo en tanto que tal, puesto que modifica únicamente la manera en que se efectúa el trabajo, no afecta al estatus laboral del trabajador. La negativa de un empleado a teletrabajar no es, en sí, un motivo de rescisión de la relación laboral ni de modificación de las condiciones de trabajo de este trabajador».

Es decir, el rechazo de la propuesta empresarial del cambio del lugar de la prestación laboral fuera del centro de trabajo o su retorno al mismo no ha de 
implicar la extinción de la relación laboral por tal motivo ni ningún otro perjuicio. No obstante, en un contexto de crisis económica, cabe vincular la oferta de teletrabajo a la paralización de despidos objetivos o colectivos por causas económicas, técnicas, organizativas o de producción, y su negativa conllevar la aplicación de los mismos (SAN 23 diciembre 2015, núm. 299/2015). pese a las dudas suscitadas en torno a la voluntariedad requerida.

Al respecto, los Tribunales han declarado que no cabe modificar (en uno u otro sentido) esta condición laboral a través del art. 41 ET (STS 11 abril 2005, Rec. núm. 143/2004), en tanto transformaría la naturaleza del vínculo. Se cercena así una posible vía de adaptación mediante la flexibilidad interna a las futuras necesidades de las prestaciones de servicios en un contexto de alta incertidumbre económica y sanitaria.

$\mathrm{El}$ art. 13.2 ET parece exigir el acuerdo de ambas partes para revertir el anterior pacto aunque no mencione dicha posibilidad. En cambio, el Acuerdo Europeo permite establecer las modalidades de reversibilidad "por acuerdo individual o colectivo", aun cuando parece dudoso que el convenio colectivo pueda eximir de la voluntad individual concurrente (Tascón López, 2020), y menos dejarlo al arbitrio exclusivo del empleador.

Aun anticipando conceptos y condiciones examinados con posterioridad, el acuerdo que establezca el teletrabajo debería recoger los siguientes extremos (NTP 412): lugar de trabajo, jornada y distribución horaria, métodos de trabajo, formación necesaria, retribución específica en caso de existir, reversibilidad de la situación, equipo de trabajo y mantenimiento, asunción de los costes, seguros, protección de datos y confidencialidad, a los que cabe añadir aquellas medidas destinadas a cumplir los requerimientos en materia de ciberseguridad.

\section{Igualdad de derechos con la prestación de servicios presencial}

$\mathrm{El}$ art. $13 \mathrm{ET}$, al igual que el AMET, reconoce y equipara en derechos a los trabajadores a distancia y los presenciales, salvo aquéllos «que sean inherentes a la realización de la prestación laboral en el mismo de manera presencial». El Acuerdo Marco Europeo dispone que esta igualdad derechos entre trabajadores ordinarios y a distancia se refleje tanto en la asignación de carga de trabajo (art. 4), como en unas mismas políticas de evaluación y valoración (art. 10).

Esta igualdad de derechos aparece destacada en materia salarial (Sierra Benítez, 2016: 224): «el trabajador tendrá derecho a percibir, como mínimo, la retribución total establecida conforme a su grupo profesional y funciones» (art. 13.3 
ET). Tanto el salario base como los complementos no pueden experimentar supresión ni disminución por llevar a cabo sus tareas a distancia. Es más, del literal del precepto cabe deducir la posibilidad de una mayor retribución a este tipo de empleados (originada en el ahorro de costes empresariales y su transmisión a quien presta servicios en remoto), siempre y cuando no suponga una discriminación vetada por el ordenamiento jurídico respecto a los presenciales.

Vinculado a la retribución, aun cuando no tenga naturaleza salarial, resulta necesario aquilatar en cada caso cómo se van a afrontar los gastos ocasionados por el teletrabajo (tanto aquellos invertidos en las herramientas necesarias como los gastos fijos de electricidad, red, etc.), bien la empresa proporciona los equipos de trabajo o bien se reembolsan o, en fin, se compensa el mayor consumo de los suministros corrientes del domicilio. No cabe olvidar en este punto cómo el empresario experimentará también un ahorro de costes respecto al centro de trabajo, que incluso podría llegar a desaparecer y que, en todo caso, experimentará una reducción de tamaño y/o de consumos fijos (Tascón López, 2020).

$\mathrm{Al}$ respecto, se pronuncia el Acuerdo Marco Europeo, que en su punto 7 establece que, «como regla general, el empresario está encargado de facilitar, instalar y mantener los equipamientos necesarios para el teletrabajo regular, salvo si el teletrabajador utiliza su propio equipo. Si el teletrabajo se realiza regularmente, el empresario cubre los costos directamente originados por este trabajo, en particular los ligados a las comunicaciones. El empresario dotará al teletrabajador de un servicio adecuado de apoyo técnico. Si el teletrabajo se realiza regularmente, el empresario cubre los costos directamente originados por este trabajo, en particular los ligados a las comunicaciones. El empresario dotará al teletrabajador de un servicio adecuado de apoyo técnico. El empresario tiene la responsabilidad, conforme a la legislación nacional y a los convenios colectivos, en lo referente a los costes ligados a la pérdida o a los desperfectos de los equipos y de los datos utilizados por el teletrabajador. El teletrabajador cuidará los equipamientos que le han confiado; no recogerá ni difundirá material ilícito vía internet».

\section{La imprescindible formación para el teletrabajo}

La formación de los teletrabajadores resulta vital en un doble sentido: por un lado, respecto a aquella destinada a seguir avanzando en su carrera profesional, teniendo las mismas oportunidades que el resto de sus compañeros que prestan servicios en el centro de trabajo físico y ligada a la promoción profesional; por otro, cuanta requiera el correcto desempeño de la relación laboral a distancia, con la finalidad de cumplir los requerimientos del trabajo desempeñado y no ser objeto de un despido por falta de adaptación a las modificaciones técnicas operadas en su puesto (De Castro Mejuto, 2016): 95), por ejemplo. 
Respecto a la primera, el art. 13.3 ET exige que el empresario establezca los «medios necesarios para asegurar el acceso efectivo de estos trabajadores a la formación profesional para el empleo, a fin de favorecer su promoción profesional». La finalidad del precepto parece clara, tratando de evitar así que el relativo aislamiento al que pueden verse abocados estos peculiares operarios termine por degenerar en su obsolescencia profesional (Tascón López, 2020) y evitar con ello que dicho trabajador sufra una pérdida de habilidades y competencias derivada de la propia distancia con los centros logísticos y organizativos de la empresa (Blázquez Agudo, 2012: 33). De nuevo, el Acuerdo Marco Europeo precisa más el derecho a la formación, ante el temor a caer en el olvido para futuros ascensos al no estar físicamente en el centro de trabajo: «los teletrabajadores tienen el mismo acceso a la formación y a las posibilidades de carrera profesional que trabajadores similares que realizan su tarea en los locales del empresario, y están sujetos a las mismas políticas de evaluación que los demás trabajadores» $\mathrm{o}$ que se diseñe la formación sin tener en cuenta las necesidades de quien no acude físicamente al centro de trabajo.

Respecto a la segunda, y frente al silencio del precepto estatutario, el Acuerdo Europeo precisa que estos trabajadores tienen derecho a recibir una formación adecuada "para utilizar el equipo técnico a su disposición y sobre las características de esta forma de organización del trabajo». De hecho, avanza un paso más (necesario) y apunta la necesidad de dar oportuna formación tanto al supervisor de los teletrabajadores como a sus compañeros de trabajado para adecuarse a esta forma de trabajo y saber gestionarla. Punto de suma trascendencia si se desea configurar el teletrabajo como modalidad de trabajo digno y decente, sin someter a quienes lo lleven a cabo a explotación tecnológica fundada en cumplimiento de objetivos excesivo o rechazada por incomprendida.

Desde luego, ninguna de las normas precisa, ni puede hacerlo, el contenido, la modalidad o la duración de la formación requerida, dependerá de cada puesto y sector y su desarrollo compete, al menos a grandes rasgos al convenio colectivo de aplicación.

Aun cuando la distancia física no debería suponer falta de información respecto a las oportunidades de reversión del teletrabajo, la promoción profesional y/o la existencia de vacantes en la empresa, la norma impone la obligación de la empresa de informar sobre las vacantes presenciales existentes, aun cuando no suponga un derecho preferente para acceder a tales plazas. La norma solo alude a la finalidad de "posibilitar la movilidad y promoción», esto es, habilita un cauce para que los teletrabajadores estén informados de las vacantes y de los procesos selectivos para proveer dichas vacantes $y$, por tanto, puedan concurrir en igualdad de condiciones (sin quedar olvidados o postergados) respecto a los presenciales (De Castro Mejuto, 2016: 95). 


\section{Interrelación entre trabajo flexible y teletrabajo}

La fórmula del teletrabajo no afecta directamente a la duración de la jornada, aun cuando sí puede modular ciertos aspectos tales como el horario, esto es, los momentos exactos en que la prestación es debida (Suárez Quiñones, 2016: 133), dado que en la mayor parte de las ocasiones se traduce teletrabajo como trabajo flexible a distancia (y en algunas ocasiones como trabajo perpetuo a distancia).

$\mathrm{Al}$ respecto, no cabe duda alguna respecto a la aplicación del nuevo art. 34.9 ET, incorporado por el art. 10 del RD-Legislativo 8/2019, de 8 de marzo, el cual ordena a la empresa garantizar «el registro diario de jornada, que deberá incluir el horario concreto de inicio y finalización de la jornada de trabajo de cada persona trabajadora, sin perjuicio de la flexibilidad horaria... Mediante negociación colectiva o acuerdo de empresa o, en su defecto, decisión del empresario previa consulta con los representantes legales de los trabajadores en la empresa, se organizará y documentará este registro de jornada. La empresa conservará los registros a que se refiere este precepto durante cuatro años y permanecerán a disposición de las personas trabajadoras, de sus representantes legales y de la Inspección de Trabajo y Seguridad Social».

En cuanto al concreto método o herramienta que llevará a cabo la recogida de datos de los teletrabajadores, necesariamente habrá de realizarse de forma telemática y remota, pero de forma objetiva y no modificable, a través de los propios instrumentos informáticos que se utilizan para el desarrollo de la prestación (Tascón López, 2020).

El registro de la jornada está destinado a evitar la fijación del trabajo por objetivos — "destajo tecnológico» (Sierra Benítez, 2016: 211) — o la perpetua disponibilidad («presentismo digital»). Pero sigue siendo posible el diseño del teletrabajo flexible, siempre y cuando el tiempo trabajado quede registrado, siendo relativamente sencillo técnicamente (Thibault Aranda, 2020).

Así lo reconoce algún pronunciamiento judicial, al entender que «solamente si la empresa ha establecido pautas claras sobre tiempo de trabajo respetuosas con la regulación legal y convencional sobre jornada y descansos y si además establece, de acuerdo con el trabajador, instrumentos de declaración y control del tiempo de trabajo a distancia o en el domicilio, sería posible admitir que una conducta del trabajador en el interior de su domicilio en vulneración de dichas pautas y omitiendo los instrumentos de control empresarial pudiera dar lugar a exceptuar el pago de las correspondientes horas y su cómputo como tiempo de trabajo. Pero en ausencia de esas pautas y criterios y de unos mínimos instrumentos de control no puede admitirse tal exceptuación, que sería equivalente a crear un espacio de total impunidad y alegalidad en el trabajo a distancia y en el domicilio" (STSJ Castilla y León/Valladolid 3 febrero 2016, Rec. 
núm. 2229/2015). También los acuerdos más recientes al respecto contemplan las medidas de aplicación a este colectivo (tal sucede con el Acuerdo de registro de jornada y desconexión digital de Deutsche Bank, SAE de 4 de diciembre de 2019).

De igual modo, parece no solo aconsejable sino necesario precisar aspectos relativos a las horas extraordinarias, pues ha de contemplarse si son posibles los excesos temporales de prestación por encima de la habitual y, en su caso, a qué régimen jurídico se someten (Monereo Pérez y Moreno Vida, 2005: 202), el trabajo nocturno o el disfrute de los descansos y vacaciones para lograr su efectivo disfrute y una real desconexión del trabajador (Tascón López, 2020). La flexibilidad antes apuntada coadyuva a eludir su control por el empresario y por la Inspección en último término.

\section{La desconexión digital en el teletrabajo}

El reverso del tiempo de trabajo es el derecho a los descansos mínimos establecidos legal o convencionalmente. De acuerdo con el TJUE el empresario tiene el deber de garantizar que el trabajador, en cualquier caso, o con cualquier tipo de relación laboral, debe atenderse su derecho al descanso efectivo (en la jurisprudencia comunitaria baste destacar STJUE C-397/2001, de 5 de octubre de 2004, asunto Pfeiffer y otros o STJUE C-151/2002, de 9 de septiembre de 2003, asunto Jaeger), aun cuando no defina qué debe entenderse por tal «descanso efectivo» (Rodríguez Rodríguez, 2019: 148).

Sin embargo, la tecnología elimina las coordenadas de tiempo y lugar, y difumina las fronteras entre trabajo y descanso, hasta el punto de provocar una conexión perpetua. Por tal motivo, es necesario apuntalar una política de prevención de riesgos laborales que blinde ese derecho al descanso en forma de desconexión (país pionero en su regulación ha sido Francia con la denominada, por la ministra de Trabajo que la promovió, Ley El Khomri que introduce en el artículo L. 2242-8 del Código de Trabajo francés dicho derecho, aun formulado de forma vaga), estrechamente ligado con el supuesto de la llamada «fatiga informática» (Baylos Grau, 2019), de conformidad con lo previsto en el art. 88 LO 3/2018 («los trabajadores y los empleados públicos tendrán derecho a la desconexión digital a fin de garantizar, fuera del tiempo de trabajo legal o convencionalmente establecido, el respeto de su tiempo de descanso, permisos y vacaciones, así como de su intimidad personal y familiar»).

Este artículo encomienda a la negociación colectiva, aunque no forme parte del contenido mínimo de los convenios (Tascón López, 2018: 45) las modalidades de ejercicio de este derecho. En el fondo, la desconexión digital supone una herramienta para articular la concreta vía para reconocer el derecho al descanso 
en un mundo hiperconectado, que ha de atender a la naturaleza y objeto de la relación laboral, y potenciar el derecho a la conciliación de la actividad laboral y la vida personal y familiar. En este sentido, y con particular mención al trabajo a distancia, continúa el precepto, el empleador, previa audiencia de los representantes de los trabajadores, ha de elaborar «una política interna dirigida a trabajadores, incluidos los que ocupen puestos directivos, en la que definirán las modalidades de ejercicio del derecho a la desconexión y las acciones de formación y de sensibilización del personal sobre un uso razonable de las herramientas tecnológicas que evite el riesgo de fatiga informática. En particular, se preservará el derecho a la desconexión digital en los supuestos de realización total o parcial del trabajo a distancia así como en el domicilio del empleado vinculado al uso con fines laborales de herramientas tecnológicas».

La razón de la mención particular radica en la interconexión entre teletrabajo y trabajo flexible, actuando la desconexión digital a modo de frontera que delimita este del descanso semanal, interjornadas o las propias vacaciones.

Hasta el momento, y debido al escaso tiempo transcurrido no resulta fácil encontrar ejemplos que sirvan de guía, pues los convenios que han empezado a regular la desconexión realizan un simple reconocimiento del derecho de forma indeterminada, vaga, falta de concreción, meramente programática o sometido a la inexistencia de "fuerza mayor» (Igartúa Miró, 2019: 80), sin concretar el deber de abstención del empresario y sin medios e instrumentos para hacerlo efectivo el derecho (García Jiménez, 2019: 172), simplemente dependiente de la «voluntariedad» del trabajador de no contestar correos o mensajes.

Mejores prácticas al respecto, y como muestra, resultan cuantos establecen la obligación de «no enviar» en lugar de no contestar (art. 40 Resolución de 19 de marzo de 2019, de la Dirección General de Trabajo, por la que se registra y publica el Convenio colectivo de Zurich Insurance PLC, Sucursal en España, Zurich Vida, Compañía de Seguros y Reaseguros, SA y Zurich Services, AIE. BOE núm. 76, de 29 de marzo de 2019); se impide técnicamente recibir comunicación del trabajo; optan por la retirada del aparato facilitado por la empresa (Mella Méndez, 2016: 11), por la desconexión total y automática, bloquean el acceso a herramientas digitales, formulan periodos de siesta digital o toque de queda digital o fijan una franja horaria en la que exclusivamente se puede conectar tras la jornada (Igartúa Miró, 2019: 81).

\section{El control empresarial cuasi absoluto de la persona teletrabajadora}

Las dudas que planteaba el trabajo a distancia en tiempos pretéritos respecto a las posibilidades de control del empresario de la actividad productiva de los empleados quedan despejadas merced a las infinitas posibilidades brindadas 
por la tecnología utilización de seguidores URL, que permiten rastrear los movimientos de los trabajadores en la red, tecnología ASP que conoce la ubicación de los trabajadores mediante el teléfono móvil, sniffers o identificadores de radiofrecuencia RFID (Cuadros Garrido, 2018: 449). No solo rastrear, sino que los datos masivos generados por la persona teletrabajadora van a permitir su tratamiento a través de algoritmos y programas de inteligencia artificial, dejando a quien teletrabaja en situación de completa "transparencia», no solo respecto a su labor, sino a su personalidad íntima.

$\mathrm{Al}$ respecto, nada en particular recoge el art. 13 ET, pero el Acuerdo Marco sí dispone que «el empresario debe respetar la vida privada del teletrabajador. Si existe un medio de vigilancia, deberá ser proporcionado al objetivo e introducirse con arreglo a la Directiva 90/270/CEE relativa a las pantallas de visualización».

Por su parte, los sujetos negociadores han de empezar a regular el amplio campo que les seńala el art. 87.3 LO 3/2018: «los empleadores deberán establecer criterios de utilización en los distintos lugares de los dispositivos digitales respetando en todo caso los estándares mínimos de protección de su intimidad de acuerdo con los usos sociales y los derechos reconocidos constitucional y legalmente» $\mathrm{y}$ «en su elaboración deberán participar los representantes de los trabajadores». De prestar servicios vía telemática, cada vez en mayor medida y con mayor incidencia el empresario tiene a su disposición de múltiples instrumentos de control y vigilancia (más o menos invasivos) necesitados de acotamiento y transparencia en su uso y finalidad por la negociación colectiva (Álvarez Cuesta, 2019: 110) y de un cambio de enfoque, prioritariamente asentado sobre una suerte de presunción de intromisión legítima del empleador en la confidencialidad de los datos y en la esfera privada de los trabajadores (Baylos Grau, 2019).

Hasta el momento, las experiencias con las que se cuenta en materia de control de las comunicaciones electrónicas del trabajador son muy pobres (Baylos Grau, 2019). Quizá provocado por el escaso tiempo transcurrido desde la promulgación de la ley orgánica. Como buenas prácticas cabría apuntar cuantas determinen los períodos en que los dispositivos podrán utilizarse para dichos fines privados; creación de carpetas en los sistemas de almacenamiento como ordenadores, tabletas o en la nube, donde el trabajador pueda incluir información tanto relacionada como la prestación de sus servicios como personal, asegurando que el empresario únicamente puede entrar a vigilar el primer tipo de contenido, quedando la carpeta privada excluida del control de la empresa o filtros de acceso (Blázquez Agudo, 2019) .

El uso de dispositivos tecnológicos puestos a disposición por la empresa o con contenido perteneciente a la empresa en distintos lugares no vinculados al centro de trabajo guarda relación también con la ciberseguridad de la empresa, no en vano una de las vulnerabilidades que toda organización debería tener en 
cuenta es el riesgo generado por las personas trabajadoras y más en caso de prestar servicios con sus propios medios telemáticos o del empresario pero sin contar con las debidas instrucciones.

Estos trabajadores han de actuar con la debida «diligencia informática» y utilizar adecuadamente los medios electrónicos y digitales puestos a su disposición, de acuerdo con las instrucciones y los códigos de autorregulación establecidos a tal efecto (Rodríguez-Piñero Royo, 2019), en esta ocasión no desde la perspectiva de la productividad, sino de la seguridad de la empresa respecto a su infraestructura, datos de los clientes o secretos empresariales.

\section{Prevención de riesgos laborales a distancia}

La protección de la salud y seguridad de los trabajadores a distancia siempre ha sido un obstáculo para la efectiva aplicación del teletrabajo, además de un verdadero enigma en su desarrollo práctico. El art. 13.4 ET, calificado como demasiado ambicioso y poco efectivo (Tascón López, 2020), concede a los trabajadores a distancia el derecho a una adecuada protección en materia de seguridad y salud e impone la aplicación, "en todo caso", de lo establecido en la Ley 31/1995, de 8 de noviembre, de Prevención de Riesgos Laborales, y su normativa de desarrollo.

La previsión responde a la genérica necesidad de asegurar que ningún trabajador sufra daño alguno en el desarrollo de su prestación laboral (Selma Penalva, 2016: 129), pero se enfrenta a dos dificultades encadenadas: en primer lugar, el lugar de la prestación se define de forma negativa (fuera del centro de trabajo); y en segundo, si el teletrabajo se llevara a cabo en el domicilio de la persona trabajadora, como ha sucedido durante la situación de alarma, su inviolabilidad acrecienta la desprotección.

Las soluciones en presencia, y dejando a un lado la previsión de emergencia de la autoevaluación recogida en el RD-Ley 8/2020, vienen dadas por convertir a la persona teletrabajadora en colaboradora necesaria o bien pactar qué lugares se aceptan como de prestación de servicios por entenderse plenamente seguros para el teletrabajador (Mella Méndez, 2015: 171).

En cuanto a los riesgos específicos concurrentes en el teletrabajo, además de los ergonómicos y ambientales (iluminación, temperatura, etc.), y de los derivados del uso intensivo de pantallas de visualización (RD 488/1997, de 14 de abril, sobre disposiciones mínimas de seguridad y salud relativas al trabajo con equipos que incluyen pantallas de visualización y su guía técnica de desarrollo), cabe detenerse en el examen de los riesgos psicosociales asociados al trabajo aislado y vinculado a la utilización intensiva de la tecnología. 
Ha de prestarse atención a la tecnoadicción, (necesidad de estar permanentemente conectado, al tecnoestrés, adicción al trabajo (workaholic) o a la depresión o ansiedad causadas por la soledad (NTP 412). Respecto a aquel, el elemento central definidor viene dado por el compromiso irracional establecido con el trabajo excesivo; estaría caracterizado por una excesiva dedicación laboral, por hacer de ella el único objetivo de su vida, por su desinterés por todo lo que no sea su trabajo y por su incapacidad para parar de trabajar (NTP 759), acentuado en este caso por las presiones o incentivos derivados, de un lado, de la necesidad de recursos y de otro, de los incentivos que ofrecen las empresas colaborativas para que aumentes tu participación (Álvarez Cuesta, 2017: 55).

En cuanto a la nomofobia, entendida como miedo irracional a estar sin teléfono móvil, genera una "infundada" sensación de incomunicación en el usuario cuando este no puede disponer de él y gemela al concepto de fomo, surge en estos trabajadores debido a la dependencia del móvil (o dispositivo que se trate) para trabajar.

El teletrabajo fuerza la aparición de problemas de salud física vinculados a la vida sedentaria y la falta de actividad física que casi siempre conlleva el trabajo con ordenadores, lo que puede incrementar el riesgo de accidentes cardiovasculares, obesidad, derrames cerebrales o diabetes; problemas ergonómicos u oftalmológicos derivados de la continua atención a pantallas y posturas inadecuadas; la «infoobesidad» o sobrecarga de información que debe procesarse (Álvarez Cuesta, 2019).

Frente a tales riesgos, no falta quien propone un adaptado reconocimiento médico que entrara a valorar el perfil de quien ha solicitado o se ha adherido al programa de teletrabajo de la empresa, detectando las potenciales pautas de conducta y sus previsibles consecuencias para la salud (Mella Méndez, 2015: 171). Quienes han investigado al respecto apuntan como características idóneas la flexibilidad, la adaptabilidad, la autodisciplina, la experiencia en el trabajo y con la organización, las habilidades de comunicación y sociales o la capacidad de gestionar el tiempo. También añaden las características que deben reunir los mandos: deseo de participar en el proyecto de teletrabajo; confianza en sus subordinados y en su plantilla; capacidad para organizar el trabajo; buenas capacidades de comunicación; capacidad para motivar a los trabajadores y fomentar en ellos la independencia e iniciativa; flexibilidad en el uso del tiempo; y orientado hacia la gestión de resultados y no hacia los procesos (NTP 412).

No cabe olvidar a este respecto tanto la necesidad de adaptar las condiciones cuando quienes presten servicios resulten especialmente sensibles a alguno de los riesgos laborales enumerados, como la de proporcionar una formación específica (añadida a la formación general requerida para el teletrabajo) en materia de prevención de riesgos laborales de acuerdo con la correspondiente evaluación realizada. 


\section{La configuración de los derechos colectivos en el teletrabajo}

El art. 13 ET finaliza en el párrafo 5, reconociendo a los trabajadores a distancia el ejercicio de los derechos de representación colectiva de conformidad con lo previsto a lo largo del propio Estatuto, y «a estos efectos, los trabajadores a distancia deberán estar adscritos a un centro de trabajo concreto de la empresa».

La mención expresa a la representación colectiva (como trabajadores por cuenta ajena que son) no resulta del todo superflua de atender al creciente individualismo que se promueve y alimenta en las prestaciones de servicios marcadas por la desaparición de un centro de trabajo físico y las dificultades que conllevan las relaciones colectivas en un mundo cada vez más digitalizado (al respecto, Rojo Torrecilla, 2019), donde se rompe la solidaridad necesaria para construir una negociación y puede impedir la promoción de intereses comunes y la superación de la competencia entre los propios trabajadores (Álvarez Cuesta, 2019), bien por su invisibilización, bien porque la prestación de servicios a distancia hace desaparecer los lazos de solidaridad colectiva.

En cuanto a su ubicación formal, surgen dudas (en cuanto forma y criterios) a la hora de adscribir trabajadores a un determinado centro para elegir representantes y para llevar a cabo una negociación colectiva de carácter estatutario. Como regla general de comienzo, corresponde al empresario configurar a su arbitrio las circunstancias que determinan la calificación de una unidad productiva como centro de trabajo (Álvarez Cuesta, 2019) y en este sentido, su libertad es muy amplia (Álvarez del Cuvillo, 2016: 141).

Uno primero, sería aquel en el que el trabajador realiza parte de su actividad en un único centro, hipótesis en la que el trabajador deberá ser adscrito a aquel (Pastor Martínez, 2018: 227). Si no existiera centro de trabajo, y por tanto los trabajadores no desempeñan sus servicios en uno, han de estar adscritos a un centro y entonces surge la posibilidad de agruparlos todos en uno (la elección corresponde al empresario) o diseminarlos en varios. En este sentido, sería factible la delimitación de los trabajadores adscritos a un centro por su acceso a la aplicación y su operatividad en un espacio físico determinado (Garrido Pérez, 2017: 221) para evitar que por esta vía se cercene el derecho de representación y de negociación. Algún convenio comienza a crear una ficticia circunscripción electoral al asignar a la misma a los teletrabajadores o dejar su asignación a la autonomía individual parecen consagrar posibilidades por parte de la negociación colectiva para modificar el marco legal (Ribes Moreno, 2018: 170).

También la inexistencia de un centro de trabajo físico puede dificultar la publicación del censo, de la lista de candidatos o la propia celebración de elecciones en un espacio determinado (Garrido Pérez, 2017: 224), si se encuentra distante el centro formal del lugar de prestación efectivo. Al final, todos los obstáculos enumerados relacionados con el espacio físico podrían resultar fácil- 
mente subsanables acudiendo a la tecnología, afirmación que sería osado realizar respecto a los motivados por el aislamiento y el individualismo de este tipo de prestación.

\section{El teletrabajo durante el estado de alarma causado por el COVID-19}

El RD-Ley 8/2020, en su preámbulo ya advertía cómo «con el fin de ejecutar las medidas de contención previstas por la normativa aplicable, garantizando al mismo tiempo la continuidad de la actividad empresarial y las relaciones laborales, se priorizarán los sistemas de organización que permitan mantener la actividad por mecanismos alternativos, particularmente por medio del trabajo a distancia, debiéndose facilitar las medidas oportunas para hacerlo posible. Para ello, se facilitan los trámites previstos por la normativa aplicable». Por ello, su art. 5 señala el carácter preferente del trabajo a distancia con los objetivos prioritarios de garantizar que la actividad empresarial y las relaciones de trabajo se reanuden con normalidad tras la situación de excepcionalidad sanitaria. "En particular, se establecerán sistemas de organización que permitan mantener la actividad por mecanismos alternativos, particularmente por medio del trabajo a distancia, debiendo la empresa adoptar las medidas oportunas si ello es técnica y razonablemente posible y si el esfuerzo de adaptación necesario resulta proporcionado. Estas medidas alternativas, particularmente el trabajo a distancia, deberán ser prioritarias frente a la cesación temporal o reducción de la actividad» (Beltrán de Heredia Ruiz, Ignasi, 2020c).

De dicho precepto parece desprenderse la obligación de la empresa de adoptar las medidas oportunas (teletrabajo) si es técnica y razonablemente posible y el esfuerzo de adaptación es proporcionado «desde un punto de vista de costes u organización» (Ginès i Fabrellas, 2020), por tanto, el deber queda supeditado a los mencionados condicionantes. Por tanto, «aparentemente no se está reconociendo un derecho unilateral» de la persona trabajadora a esta modalidad (Beltrán de Heredia Ruiz, 2020: 50). Si el cambio es posible, esta opción es prioritaria frente a los expedientes de regulación temporal de empleo (Ginès i Fabrellas, 2020) y no cabe la imposición del permiso obligatorio (CC.OO., 2020: 23).

En cuanto hace al requisito de técnico, excluye, obviamente, todas las prestaciones personales que no puedan realizarse bajo ningún concepto a distancia». En cambio, cuando esta imposibilidad técnica solo abarque parte de sus funciones, sobre todo en los supuestos de polivalencia funcional, parece evidente que esta obligación subsistirá para aquellas funciones que sí admitan técnicamente dicha posibilidad» (Calvo Gallego, 2020).

La formulación del teletrabajo y su reversión condicionado a un acuerdo de voluntades hace cuestionar la nota de la voluntariedad, en un primer momento, 
para su adopción, y en un segundo, para su reversión. Parece claro que, en este contexto, «la persona trabajadora no puede imponer el teletrabajo» (CC.OO., 2020: 77), y no faltan opiniones divergentes, bien porque entienden exigible el acuerdo aún en esta situación (Ginès i Fabrellas, 2020); bien porque consideran que se ha producido una «suspensión temporal del régimen ordinario de derechos y obligaciones legales y/o convencionales y/o contractuales» y el empleador puede disponer unilateralmente de la modalidad de prestación de servicios, a modo de «rebus sic statibus provisional» (Falguera Baró, 2020: 128); o bien por considerar esta opción obligatoria para ambos (Casas Baamonde y RodríguezPiñero y Bravo-Ferrer, 2020: 329).

La solución a las posibilidades de reversión es diferente conforme a la respuesta anterior. De exigirse voluntad de ambas partes para su adopción, se requiere la misma para su vuelta a la presencialidad, salvo que se entienda condicionado el acuerdo alcanzado a la propia situación de pandemia; en cambio, de considerar el teletrabajo excepcionalmente impuesto, una vez transcurridos los plazos, la persona trabajadora ha de volver a la presencialidad y tiene derecho a hacerlo (salvo acuerdo novatorio). También cabe una posición intermedia, a aplicar caso por caso: al ser una medida unidireccional, «dirigida a interrumpir el tránsito de la actividad a la inactividad empresarial». Lo que podría ser determinante porque, si no se da esta situación material previa, podría cuestionarse la posibilidad de aplicar este precepto y en esas circunstancias, «sería controvertido afirmar que el trabajo a distancia mantiene la preferencia» (Beltrán de Heredia Ruiz, 2020b).

El escollo existente en materia preventiva se intenta salvar entendiendo cumplida la obligación de efectuar la evaluación de riesgos, con carácter excepcional, a través de una autoevaluación realizada voluntariamente por la propia persona trabajadora en aquellos sectores, empresas o puestos de trabajo en las que no estuviera prevista hasta el momento (art. 5 RD-Ley 8/2020). Al efecto, las empresas han debido proporcionar la debida formación e instrucciones para llevarla a cabo. Con todo, no se despeja la duda sobre la respuesta empresarial frente a una negativa de la persona trabajadora a realizarla, si bien la misma no podría bloquear el recurso al teletrabajo en las coordenadas existentes (Cruz Villalón, 2020: 410).

Donde sí estuviera implantada esta modalidad, la evaluación ha debido ser realizada previamente, dentro de la obligación preventiva habitual de la organización. En todo caso, y «sin perjuicio de la redacción del precepto, la autoevaluación de la persona trabajadora no exime a la empresa de toda obligación de evaluación de riesgos, sino solamente de la evaluación inicial de riesgos. Es decir, la empresa deberá realizar una evaluación de los riesgos en atención a dicho informe de autoevaluación para garantizar la seguridad y salud de las personas durante el periodo de teletrabajo» (Ginès i Fabrellas, 2020). "En consecuencia, 
no existe exoneración de la obligación empresarial de proporcionar la información necesaria (art. $18 \mathrm{LPRL}$ ) o proceder, en su caso, a dar la formación correspondiente (art. 19 LPRL)» (Falguera Baró, 2020: 128).

La duración de esta situación, destinada a garantizar la protección de las personas trabajadoras y seguir atendiendo a las necesidades de conciliación de la vida laboral y familiar en el contexto de la crisis del COVID-19 (por todos, Rodríguez Escanciano, 2020), se prorroga durante tres meses (dos meses +1 mes) una vez finalizado el estado de alarma: «de acuerdo con lo previsto en el segundo párrafo de la disposición final décima, del Real Decreto-ley 8/2020, de 17 de marzo, se prorroga lo establecido en los artículos 5 y 6 de dicha norma. El contenido de estos artículos se mantendrá vigente durante los dos meses posteriores al cumplimiento de la vigencia prevista en el párrafo primero de la disposición final décima del Real Decreto-ley 8/2020, de 17 de marzo, modificado por la Disposición Final 1.17 del Real Decreto-ley 11/2020, de 31 de marzo», aun cuando, «en atención a las circunstancias, cabrán prórrogas adicionales por parte del Gobierno de lo establecido en el presente precepto» (art. 15 RDLey 15/2020).

De este modo, el art. 3 de la Orden SND/399/2020, de 9 de mayo, para la flexibilización de determinadas restricciones de ámbito nacional, establecidas tras la declaración del estado de alarma en aplicación de la fase 1 del Plan para la transición hacia una nueva normalidad, sigue considerando preferente mantener los medios no presenciales de actividad productiva: «siempre que sea posible, se fomentará la continuidad del teletrabajo para aquellos trabajadores que puedan realizar su actividad laboral a distancia». De nuevo, el precepto condiciona su continuidad a la posibilidad efectiva de la misma y a su indeterminado «fomento».

Una de las posibilidades para su fomento quizá resida en el Plan Acelera diseñado por el RD-Ley 8/2020, con el fin de establecer medidas de apoyo para acelerar el proceso de digitalización de las pymes que pasan por el asesoramiento y la formación, las soluciones tecnológicas y el apoyo financiero.

\section{A modo de conclusión: vías para la aplicación en el futuro del teletrabajo en la empresa}

El teletrabajo ha sido un recurso excepcional destinado a la protección de la salud y la seguridad de los trabajadores presenciales al tiempo que aseguraba el mantenimiento de la actividad productiva en la empresa durante la pandemia causada por el COVID19, y todo parece indicar que la rápida adaptación al mismo (con sus fallos) hace que la cultura del presentismo esté en franco declive y muchas empresas decidan continuar con el trabajo a distancia. También 
contribuye, sin duda, que «el cambio repentino al trabajo digital a distancia, de la noche a la mañana y en masa, tiene el potencial de acelerar los cambios en la forma en que se realiza el trabajo» (Rojo Torrecilla, 2020: 391).

A ello se une la expansión sin precedentes de la tecnología y su aplicación a las más variadas prestaciones de servicios. Por todos esos motivos, unidos a las ventajas ya citadas, parece que el teletrabajo (en la regulación actual o en otra más acabada) va a experimentar un gran crecimiento, analizándose a continuación las posibles vías articulables para transformar un trabajo presencial en trabajo a distancia (y viceversa).

A. La primera de ellas en los términos examinados, es el acuerdo inicial (al venir ya configurada así la relación laboral) o sobrevenido, pero en todo caso precisa la voluntad concurrente de empresario y persona trabajadora. Respecto a esta vía, quizá el experimento sufrido puede llevar a facilitar el acuerdo en uno u otro sentido, pero ha de tenerse en cuenta los puntos críticos apuntados respecto al mismo, haciendo deseable una mayor regulación convencional que dote de un adecuado marco a su desarrollo y evite el fácil recurso al teletrabajo invisible. Es más, no falta quien apunta a que el acuerdo exigido, en el futuro ha de ser colectivo (Cruz Villalón, 2020: 417).

B. La segunda supone una manifestación del derecho a la conciliación ya anunciado, pues tradicionalmente se ha configurado el teletrabajo como herramienta capaz de favorecer la misma (Autores Varios, 2012 y Eurofound, 2020). Y dicha posibilidad sigue presente en el art. 34.8 ET, el cual reconoce el derecho de las personas trabajadoras a solicitar las «adaptaciones de la duración y distribución de la jornada de trabajo, en la ordenación del tiempo de trabajo y en la forma de prestación, incluida la prestación de su trabajo a distancia, para hacer efectivo su derecho a la conciliación de la vida familiar y laboral».

También la Directiva 2019/1158 del Parlamento europeo y del Consejo de 20 de junio de 2019 relativa a la conciliación de la vida familiar y la vida profesional de los progenitores y los cuidadores, y por la que se deroga la Directiva 2010/18/UE del Consejo, aún no incorporada a nuestro acervo legislativo, contempla el recurso al teletrabajo como respuesta a las necesidades de conciliación. Así, establece como derechos fórmulas de trabajo flexible para los trabajadores que sean progenitores o cuidadores, entendiendo por tales la «posibilidad de los trabajadores de adaptar sus modelos de trabajo acogiéndose a fórmulas de trabajo a distancia, calendarios laborales flexibles o reducción de las horas de trabajo» (art. 3), supeditadas, en su caso, a períodos de trabajo anterior o a una antigüedad que no podrá ser superior a seis meses (art. 9.4). Asimismo, prevé el recurso a estas fórmulas flexibles (incluido el teletrabajo) para el 
disfrute del permiso de paternidad, del permiso parental (cada trabajador tenga un derecho individual a disfrutar de un permiso parental de cuatro meses que debe disfrutarse antes de que el hijo alcance una determinada edad, como máximo ocho años) y para el cuidado de familiares. El art. 9 desarrolla este recurso al teletrabajo: así, los empleadores estudiarán y atenderán las solicitudes de acogerse a fórmulas de trabajo flexible en un plazo razonable de tiempo, teniendo en cuenta tanto sus propias necesidades como las de los trabajadores. Pese a que no establece su concesión, la denegación de la petición cursada o su aplazamiento requiere justificación. En cuanto a su reversión, sí configura un derecho de la persona trabajadora a volver a su modelo de trabajo original al término del período acordado, incluso tendrá derecho a solicitar volver a su modelo de trabajo original antes de que finalice el período acordado siempre que lo justifique un cambio en las circunstancias y dicha solicitud será estudiada por el empleador teniendo en cuenta tanto sus propias necesidades como las de los trabajadores.

Como muestra señera de Derecho comparado, la Ley portuguesa $120 / 2015$, de 1 de septiembre, pretende reforzar los derechos de los padres trabajadores a conciliar su vida laboral y familiar por la vía del teletrabajo. Así, dicha Ley da nueva redacción al art. 166.3 Código de Trabalho, que pasa a establecer que, «aparte de las situaciones referidas en el número anterior (ser víctima de violencia doméstica), el trabajador con hijo de edad hasta 3 años tiene derecho a ejercer la actividad en régimen de teletrabajo, cuando este sea compatible con la actividad desempeñada y la entidad patronal disponga de recursos y medios para tal efecto». Los presupuestos objetivos exigidos para el ejercicio de tal derecho por parte del trabajador son dos: uno, la compatibilidad de la actividad laboral de aquel con el nuevo modo de trabajar a distancia, pues, en caso contrario, la medida no tiene sentido, en cuanto el trabajador debe seguir siendo productivo para la empresa; el otro, que esta última disponga de recursos y medios para el desarrollo del teletrabajo (Mella Méndez, 2017).

Al tiempo, resulta preciso advertir de las aristas que presenta su utilización en un contexto como el actual, capaz de convertir este instrumento en un refugio para mujeres con responsabilidades familiares, lo que puede llegar a constituir un foco de discriminación y menoscabo de las condiciones de trabajo por razón de sexo (Villalba Sánchez, 2016: 74). Ante este panorama, la corresponsabilidad se dibuja como imprescindible para evitar que nazca otro nicho de trabajo precarizado y feminizado.

C. No cabe obviar la posibilidad de aplicar el procedimiento de modificación sustancial, de entender el teletrabajo como un sistema de trabajo (parece apuntarlo Falguera Baró, 2020), quizá para configurar este recurso de modo parcial, a modo de "flexibilidad tecnológica» (Lahera 
Forteza, 2020: 428), cumpliendo lo previsto en el punto 11 del Acuerdo Marco Europeo, que reconoce a los representantes de los trabajadores el derecho a ser informados y consultados sobre la introducción del teletrabajo en la empresa.

Bien es cierto que hasta el momento parece estar vetada la vía apuntada (y así parece señalarse también en la "Consulta pública previa a la elaboración de un proyecto normativo consistente en la modificación y elaboración de las condiciones para prestar trabajo por cuenta ajena a distancia»), aun cuando sí es posible articular el teletrabajo como una medida de flexibilidad interna (acordada) en lugar de acudir a despidos por causas objetivas o colectivos. De este modo, se mantiene el salario, las cotizaciones, el empleo y supone un ahorro de costes para el empresario y para la Seguridad Social.

D. A semejanza de lo previsto en el art. 34.2 ET para el tiempo de trabajo y la posibilidad de pactar su distribución irregular (dejando en manos del empresario en caso contrario la distribución del $10 \%$ de la jornada anual) cabría una reforma legal que flexibilizada la localización de la prestación de servicios de un modo semejante. Ahora bien, en tal caso, parecería preceptivo articular un contrapeso colectivo a dicha opción o asegurar el mantenimiento del empleo de recurrir a la misma.

E. No cabría plantear, ni siquiera teóricamente, el recurso a la movilidad geográfica cuando el empresario decida cambiar el lugar de la prestación de servicios al domicilio del empleado y seguir el procedimiento previsto para el traslado, en tanto se requiere cambio de residencia de la persona trabajadora (art. 40 ET).

Tal y como señala la «Consulta pública previa a la elaboración de un proyecto normativo consistente en la modificación y elaboración de las condiciones para prestar trabajo por cuenta ajena a distancia»: "el impacto real de estas formas de prestación u organización del trabajo se ha visto incrementada de manera exponencial por el impacto de la pandemia del COVID-19, lo que ha puesto de manifiesto sus ventajas y debilidades, así como la necesidad de que se aborde su regulación desde un marco jurídico de seguridad, certeza y transparencia».

Por ello, y ante las múltiples sendas por las que puede discurrir el futuro del teletrabajo y su difusión, es preciso volver a insistir en la necesidad de la proyectada regulación legal (y convencional) no solo del teletrabajo, sino de distintas modalidades de prestación de servicios que recurran de forma parcial al trabajo fuera del centro, capaz de sortear los escollos planteados en materia de prevención de riesgos, suplido de gastos, formación debida, control empresarial, brecha de género, jornada de trabajo o exclusión de determinados territorios por falta de la tecnología necesaria. 


\section{Bibliografía}

Autores varios (2012): El libro blanco del teletrabajo en España. Del trabajo a domicilio a los e-workers. Un recorrido por la flexibilidad espacial, la movilidad y el trabajo remoto, Madrid: Fundación Más Familia.

Álvarez Cuesta, Henar (2018): «La lucha contra los “falsos autónomos” en la prestación de servicios vía app. El caso "deliveroo"», Iuslabor, 2.

Álvarez del Cuvillo, Antonio (2016): «El centro de trabajo como unidad electoral: un concepto jurídico indeterminado en un contexto de cambio organizativo», NREDT, 188.

Álvarez Cuesta, Henar (2017): «La prevención de riesgos laborales en la economía colaborativa: los e-nómadas», en Tiempos de cambio y salud mental de los trabajadores, Albacete: Bomarzo.

Álvarez Cuesta, Henar (2019): «Lugar único, múltiple y ausencias de lugar a partir de la negociación colectiva", Documentación Laboral, 118.

Álvarez Cuesta, Henar (2019b): «La ciberseguridad en la empresa: una aproximación desde el Derecho del Trabajo", Revista de Derecho de las Relaciones Laborales, 7.

Baylos Grau, Antonio (2019): «El papel de la negociación colectiva en la ley de protección de datos personales y garantía de derechos digitales en España», Labour and Law Issues, 5.

Beltrán de Heredia Ruiz, Ignasi (2020): "COVID-19 y medidas sociolaborales de emergencia (RDLey 6/20, 7/20, 8/20, 9/20, 10/20, 11/20, 12/20 y 13/20)», Iuslabor, 1 .

Beltrán de Heredia Ruiz, Ignasi (2020b): "Teletrabajo y desescalada: ¿en qué medida puede exigirse el reingreso al centro de trabajo?», Una mirada crítica a las relaciones laborales, 27 de mayo de 2020.

Beltrán de Heredia Ruiz, Ignasi (2020c): «COVID-19: Caminando en la niebla y el Derecho laboral de excepción", Una mirada crítica a las relaciones laborales, 4 de junio de 2020.

Blázquez Agudo, Eva (2012): El derecho a la formación para el empleo: el art. 23 ET, Pamplona: Aranzadi.

Blázquez Agudo, Eva (2019): «Novedades laborales en la nueva Ley orgánica de protección de datos», Trabajo y Derecho, 50.

Calvo Gallego (2020): «Trabajo a distancia y otras medidas alternativas», http://grupo. us.es/iwpr/covid-19-y-derecho-social/objetivos-y-principales-medidas-en-el-planolaboral/trabajo-a-distancia/.

Camas Roda, Ferrán (2018): «Tecnología y relaciones laborales: cambios en la jornada y en el lugar de trabajo. Aportaciones del Derecho Comunitario y comparado", en XXIX Jornadas Catalanas de Derecho Social: nuevas tecnologías y relaciones laborales, http://www.iuslabor.org/jornades-i-seminaris/ponencies/.

Casas Baamonde, María Emilia y Rodríguez-Piñero y Bravo-Ferrer, Miguel (2020): «Un nuevo Derecho del Trabajo en la emergencia. Las medidas laborales y de seguridad social excepcionales en el estado de alarma declarado por la crisis sanitaria de COVID-19", Derecho de las Relaciones Laborales, 4.

CC.OO. (2020): Manual de respuesta sindical en caso de ERTE, prestaciones por desempleo y otras situaciones relacionadas con el COVID-19, CC.OO.

CC.OO. (2020b): El teletrabajo desde la perspectiva de género y salud laboral, CC.OO. 
Cruz Villalón, Jesús (2020): «Teletrabajo y coronavirus: de la emergencia a la permanencia», Derecho de las Relaciones Laborales, 4.

Cuadros Garrido, M. ${ }^{a}$ Elisa (2018): Trabajadores tecnológicos y empresas digitales, Cizur Menor: Aranzadi.

De Castro Mejuto, Luis Fernando (2016): «La formación y promoción profesional en el teletrabajo", Trabajo a distancia y teletrabajo, Pamplona: Aranzadi.

De Las Heras García, Arancha (2016): El teletrabajo en Esapaña: un análisis crítico de normas y prácticas, Madrid: CEF.

Espín SÁEz, Maravillas (2014): «Los convenios de la OIT sobre el trabajo a domicilio y el trabajo a distancia», Revista del Ministerio de Empleo y Seguridad Social, 112.

Eurofound (2020): Telework and ICT-based mobile work: Flexible working in the digital age, New forms of employment series, Luxemburgo: Publications Office of the European Union.

Eurofound y OIT (2017): Working anytime, anywhere: The effects on the world of work, Ginebra: Publications Office of the European Union, Luxembourg, and the International Labour Office.

Falguera Baró, Miguel Ángel (2020): «Comentarios sobre la regulación excepcional derivada de la COVID-19 respecto al régimen contractual laboral», Ciudad del Trabajo, 30.

García JimÉNEZ, Manuel (2019): «Revolución industrial 4.0, sociedad cognitiva y relaciones laborales: retos para la negociación colectiva en clave de bienestar de los trabajadores", RTSS(CEF), 452.

García Romero, Belén (2012): El teletrabajo, Madrid: Civitas.

GArrido PÉrez, Eva (2017): «La representación de los trabajadores al servicio de las plataformas colaborativas», RDS, 80, 2017.

Ginès I Fabrellas, Anna (2020): 100 preguntas y respuestas sobre las medidas laborales ante el COVID-19, http://itemsweb.esade.edu/research/Esade-Guia-100-PreguntasRespuestas-Covid-19-FINAL-convertido.pdf.

Igartúa Miró, Teresa (2019): «El derecho a la desconexión en la Ley orgánica 3/2018, de 5 de diciembre, de protección de datos personales y garantía de los derechos digitales», RTSS(CEF), 452.

Lahera Forteza, Jesús (2020): «Flexiseguridad laboral ante la crisis del coronavirus», Derecho de las Relaciones Laborales, 4.

Leonés SALIdo, José Manuel (2000): «Razones urgentes para una regulación del teletrabajo en España", Actualidad Jurídica Aranzadi, 431.

Lousada Arochena, José Fernando (2011): «El trabajo a domicilio y el teletrabajo», Revista Técnico Laboral, 129.

Mella Méndez, Lourdes (2002): «Comentario general al Acuerdo Marco sobre teletrabajo", La Ley Digital, 4156: 7.

Mella Méndez, Lourdes (2015): "La seguridad y salud en el trabajo», en Trabajo a distancia y teletrabajo: estudios sobre su régimen jurídico en el Derecho español y comparado, Cizur Menor: Aranzadi.

Mella MÉndez, Lourdes (2016): «Nuevas tecnologías y nuevos retos para la conciliación y la salud de los trabajadores», Trabajo y Derecho, 16.

Mella Méndez, Lourdes (2017): «El trabajo a distancia como medida de flexibilidad y conciliación laboral», IUS Labor, 2.

Ministerio de Trabajo y Economía Social (2020): Guia para la actuación en el ámbito laboral en relación al nuevo coronavirus. 
Monereo Pérez, José Luis. y Moreno Vida, María Nieves (2005): El contenido de la negociación colectiva de empresa en la era de la constitución flexible del tiempo de trabajo, Valencia: Tirant lo Blanch.

NTP 412: Teletrabajo: criterios para su implantación.

NTP 759: La adicción al trabajo.

OIT (2020): Promover el empleo y el trabajo decente en un panorama cambiante, Ginebra, OIT.

ONTSI (2020): Dossier de indicadores de teletrabajo y trabajo en movilidad en España y la UE, Ministerio de Asuntos Económicos y Transformación Digital.

Pastor Martínez, Alberto (2018): «Una aproximación a la problemática de la representación colectiva de los trabajadores de las plataformas "colaborativas" y en entornos virtuales», IUS Labor, 2.

Purcalla Bonilla, Miguel Ángel y Preciado Domènech, Carlos Hugo (2013): «Trabajo a distancia vs. teletrabajo: estado de la cuestión a propósito de la reforma laboral de 2012», $A L, 2$.

Ribes Moreno, María Isabel (2018): «Representación unitaria y centro de trabajo: un modelo en crisis», Temas Laborales, 141.

Rodríguez Escanciano, Susana (2020): «Medidas para la conciliación de la vida laboral y familiar ante la emergencia sanitaria por COVID-19", Derecho de las Relaciones Laborales, 4 .

Rodríguez-Piñero Royo, Miguel (2018): «El papel de la negociación colectiva. Contenidos a afrontar, aparición de nuevas actividades y nuevas formas de trabajo», en $E l$ futuro del Trabajo: retos para la negociación colectiva, Madrid: Ministerio de Trabajo, Migraciones y Seguridad Social: 93-148.

Rodríguez-Piñero Royo, Miguel (2019): «Trabajo en plataformas: innovaciones jurídicas para unos desafíos crecientes», DP: Revista d'Internet, Dret i Politica, 28.

Rodríguez Rodríguez, Emma (2019): «La transcendencia de la disponibilidad horaria del trabajador en el contexto de las plataformas digitales», Temas Laborales, 146.

Rojo Torrecilla, Eduardo (2019): «Cambios en el mundo del trabajo. El ejercicio de los derechos colectivos en el entorno empresarial digital y la adaptación sindical», en $E l$ nuevo y cambiante mundo del trabajo. Una mirada abierta y critica a las nuevas realidades laborales, http://www.eduardorojotorrecilla.es/2019/06/cambios-en-el-mundo-deltrabajo-el.html, 3 de junio de 2019.

Rojo Torrecilla, Eduardo (2020): «Emergencia sanitaria y legislación laboral. ¿Qué respuestas a la crisis? Un primer análisis de la normativa dictada en España», Derecho de las Relaciones Laborales, 4.

Rojo Torrecilla, Eduardo (2020b): «El trabajo a domicilio (en versión clásica y moderna). La aplicación del Convenio núm. 177 y la Recomendación núm. 184 de la OIT (y referencias a la normativa española y al acuerdo europeo)", en El nuevo y cambiante mundo del trabajo. Una mirada abierta y critica a las nuevas realidades laborales, http://www.eduardorojotorrecilla.es/2020/03/el-trabajo-domicilio-en-version-clasica. html, 17 de marzo de 2020.

SAnguinetti Raymond, Wilfredo (2003): Teletrabajo y globalización en busca de respuestas al desafío de la transnacionalización del empleo, Madrid: Ministerio de Trabajo e Inmigración.

Selma Penalva, Alejandra (2016): «El accidente de trabajo en el teletrabajo. Situación actual y nuevas perspectivas», Temas Laborales, 134. 
Sempere Navarro, Antonio Vicente y Kahale Carrillo, Djamil (2013): Teletrabajo, Madrid: Francis Lefebvre.

Serrano García, Juana María (2002): «Tratamiento del teletrabajo en el Acuerdo Marco Europeo de 16 de julio de 2002», RL, 21.

Serrano García, Juana María (2009): "El teletrabajo parcial como instrumento para la conciliación en la negociación colectiva», Revisa de Derecho Social, 45.

Sierra Benítez, Esperanza Macarena (2016): «La retribución, suspensión y extinción en el teletrabajo», en Trabajo a distancia y teletrabajo, Cizur Menor: Aranzadi, pág. 224.

Solà i Monels, Xavier (2004): «El deber empresarial de protección en los supuestos de teletrabajo; contenido y alcance», en Nuevas tecnologías de la información y la comunicación y Derecho del Trabajo, Albacete: Bomarzo.

SuÁrez Quiñones, Juan Carlos (2016): «La organización del tiempo de trabajo y descanso y la conciliación en el teletrabajo», en Trabajo a distancia y teletrabajo, Pamplona: Aranzadi.

Tascón López, Rodrigo (2018): «El derecho de desconexión del trabajador (potencialidades en el ordenamiento español)», Trabajo y derecho, 41.

TAscón López, Rodrigo (2020): «Capítulo VII: El teletrabajo como forma de presente y futuro de prestación laboral de servicios. Experiencias en la negociación colectiva», en prensa.

Thibault Arana, Javier (2000): El teletrabajo, Madrid: CES.

Thibault Arana, Javier (2020): «Teletrabajo: registro horario y accidentes en el hogar», El Derecho.com, https://elderecho.com/teletrabajo-registro-horario-accidentes-hogar, 27 de marzo de 2020.

Villalba SÁnchez, Alicia (2016): «Teletrabajo y responsabilidad social empresarial», en Trabajo a distancia y teletrabajo, Cizur Menor: Aranzadi. 